\title{
Uncombined precise orbit and clock determination of GPS and BDS-3
}

\author{
Tian Zeng ${ }^{1,2}$, Lifen Sui ${ }^{1}$, Rengui Ruan ${ }^{2,3^{*}}$, Xiaolin $\mathrm{Jia}^{2,3^{*}}$ and Laiping Feng ${ }^{2,3}$
}

\begin{abstract}
There is increasing concern about the uncombined (UC) observation model in the field of global navigation satellite system (GNSS). Based on the global positioning system (GPS) and the third-generation BeiDou navigation satellite system (BDS-3), this study processed the UC precision orbit determination (POD) for single and dual systems. First, a UC observation model suitable for multi-GNSS POD was derived, and the ionospheric-free (IF) combination observation model was presented. Although the ambiguity parameters of UC and IF strategies were different after reparameterization, the difference could be removed when processing ambiguity resolution, and the equivalence was proved theoretically. To demonstrate the accuracy of BDS-3 orbits fully, the observation data of approximately 1 month were selected for determining the precise orbit for global positioning system (GPS) only, BDS-3 only, and GPS/BDS-3 systems based on the UC and IF models. The orbit precision of BDS-3 satellites was validated by using metrics, including comparison with precision products released by Wuhan University, orbit boundary discontinuity, and satellite laser ranging (SLR) residuals. The results show that the orbit accuracies of the IF and UC models are almost the same, the difference in orbits is approximately several millimeters, and the clock difference is within 0.01 ns. The GPS/BDS-3 combined solution shows better accuracy compared to other solutions. The average accuracies in the R and 3D directions are approximately 4 and $15 \mathrm{~cm}$, and the clock standard deviation is approximately $0.2 \mathrm{~ns}$ compared to external orbit product. The root mean square of SLR residuals is approximately $4 \mathrm{~cm}$.
\end{abstract}

Keywords: Precise orbit determination, GPS, BDS-3, Uncombined model, lonospheric-free combination

\section{Introduction}

In recent years, with the development of global satellite navigation system (GNSS), an increasing number of GNSSs, such as BeiDou navigation satellite system (BDS) and Galileo, can broadcast signals of at least three frequencies. Multi-frequency GNSS data processing has received widespread attention. Because the uncombined (UC) observation model is simple and unified, it has become a current focus of research. The UC model has been applied widely, such as in precision positioning (Zhang et al. 2012; Li et al. 2013), ionosphere modeling (Xiang et al. 2019), and timing (Tu et al. 2019). Many key issues, such as ambiguity resolution (AR) (Gu et al.

\footnotetext{
*Correspondence: rrg2002me@163.com; 13891907401@139.com

${ }^{2}$ State Key Laboratory of Geo-Information Engineering, Xi'an 710054,

People's Republic of China

Full list of author information is available at the end of the article
}

2015; Li et al. 2018), the third-frequency biases processing (Guo and Geng 2018; Pan et al. 2019), and ionosphere model estimation (Zhao et al. 2019), are being solved. In the field of precise orbit determination (POD), Guo (2014) and Chen (2015) successively showed the results of UC POD in their doctoral dissertations. However, the processing is time-consuming because of the huge estimated slant ionospheric parameters. Zeng et al. (2019b) compared the time costs of station-satellite elimination and epoch elimination for ionospheric parameters, and they showed that the former method can significantly decrease computing time. Strasser et al. (2019) introduced a procedure using the UC model to generate POD products. POD was performed over a 15 -year period, and the results were compared with those of the products of other analysis centers. The results showed that the product accuracy is excellent. 
BDS-3 was announced to serve global users on December 27, 2018 (Yang et al. 2019). Many scholars have evaluated the performance of BDS-3, including signal quality (Xu et al. 2019; Zhang et al. 2019; He et al. 2020), realtime kinematics (Zhang et al. 2019), precise point positioning (Zhang et al. 2019), POD (Yan et al. 2019; Zeng et al. 2019a), and clock performance (Jia et al. 2019). Li et al. (2019) showed that there is no evident systematic bias between BDS-2 and BDS-3. Guo et al. (2019) introduced the BDS-3 experimental system and service performance. Xu et al. (2019) analyzed the orbit accuracy of BDS-3 satellites. The accuracies of the 2-day overlapping orbit in the tangential $(\mathrm{T})$, normal $(\mathrm{N})$, and radial (R) directions were $5.0,2.2$, and $1.5 \mathrm{~cm}$, respectively. The root mean square (RMS) of satellite laser ranging (SLR) residuals for four BDS-3 satellites was 4-6 cm. Yang et al. (2020) analyzed the basic performance and introduced the future development of BDS-3.

However, few studies have used the UC observation model to process the POD, in particular for BDS-3 satellites, and hence, the orbit accuracy of BDS-3 needs further evaluation. In this study, the UC POD model of the GPS/BDS-3 dual system is developed, and the theoretical equivalence of ionospheric-free (IF) and UC models is deduced. Then, the BDS-3 UC POD result is presented for the first time. This work was conducted based on the software of the satellite positioning and orbit determination system (SPODS) (Ruan et al. 2014).

\section{Methodology}

GNSS observation equations for the IF and UC strategies are introduced, and the unknowns are re-parameterized. Then, the ambiguity fixing method of the UC strategy is presented, and the equivalence to the IF strategy is deduced.

\section{GNSS observation equations and reparameterization}

The raw GNSS observation equation for the carrier phase and code is

$$
\left\{\begin{array}{c}
P_{r, i}^{s}=\rho_{r}^{s}+c \delta t_{r}-c \delta t^{s}+m_{r}^{s} T_{r} \\
\quad+\gamma_{i} I_{r, 1}^{s}+B_{r, i}-B_{i}^{s}+\varepsilon_{r, i}^{s} \\
L_{r, i}^{s}=\lambda_{i} \varphi_{r, i}^{s}=\rho_{r}^{s}+c \delta t_{r}-c \delta t^{s}+m_{r}^{s} T_{r} \\
\quad-\gamma_{i} I_{r, 1}^{s}+\lambda_{i}\left(N_{r, i}^{s}+b_{r, i}-b_{i}^{s}\right)+\xi_{r, i}^{s}
\end{array}\right.
$$

where $P$ is the pseudorange measurement, and $L$ and $\varphi$ denote carrier phase measurements in meters and cycles. In addition, $s$ and $r$ denote satellite and station, $i$ denotes frequency, and $\lambda$ denotes the wavelength; $\delta t_{r}, \delta t^{S}$, and $T$ denote the receiver and satellite clock offsets and the tropospheric delay, $c$ is the speed of light, and $m$ is the mapping function of the troposphere. The first-order term of ionospheric delay at the first frequency is $I_{r, 1}^{S}$, and its coefficient is $\gamma_{i}=\left(f_{1} / f_{i}\right)^{2}$, where $f$ denotes frequency. Moreover, $B_{r, i}$ and $B_{i}^{S}$ are pseudorange time-invariant hardware delays for receiver and satellite, $b_{r, i}$ and $b_{i}^{s}$ are carrier time-invariant hardware delays for receiver and satellite. Here, $N$ is the integer ambiguity, and $\varepsilon$ and $\xi$ are the pseudorange and carrier noise, respectively.

GNSS precise clock products released by the International GNSS Service (IGS) are based on the IF combination. The following equation can be obtained:

$$
B_{r, i}-B_{i}^{s}=B_{r, I F_{12}}-B_{I F_{12}}^{s}-\frac{\gamma_{i}}{\gamma_{2}-1}\left(D C B_{r, 12}-D C B_{(2)}^{\{}\right)
$$

where $B_{r, I F_{12}}=\alpha_{12} \cdot B_{r, 1}+\beta_{12} \cdot B_{r, 2}$ and $B_{I F_{12}}^{s}=\alpha_{12} \cdot B_{1}^{s}$ $+\beta_{12} \cdot B_{2}^{s}$, and $\alpha_{12}=f_{1}^{2} /\left(f_{1}^{2}-f_{2}^{2}\right), \beta_{12}=-f_{2}^{2} /\left(f_{1}^{2}-f_{2}^{2}\right)$. Here, $B_{r, I F_{12}}$ and $B_{I F_{12}}^{s}$ denote the IF combined hardware delay of the receiver and satellite, respectively, and $D C B_{12}^{s}=B_{1}^{s}-B_{2}^{s}$ and $D C B_{r, 12}=B_{r, 1}-B_{r, 2}$ denote the differential code bias of the satellite and receiver, respectively. The IF observation model with the Taylor series expansion can be written as

$$
\left\{\begin{aligned}
P_{r, I F}^{s} & =\boldsymbol{u}_{r}^{s} \cdot\left(\boldsymbol{\Phi}\left(t_{0}, t\right)^{s} \cdot \boldsymbol{x}_{0}^{s}-\boldsymbol{x}_{r}\right)+c{\overline{\delta t_{r}}}_{r}-c \overline{\delta t^{s}} \\
& +m_{r}^{s} T_{r}+\zeta_{r, I F}^{s y s}+\varepsilon_{r, I F}^{s} \\
L_{r, I F}^{s} & =\boldsymbol{u}_{r}^{s} \cdot\left(\boldsymbol{\Phi}\left(t_{0}, t\right)^{s} \cdot \boldsymbol{x}_{0}^{s}-\boldsymbol{x}_{r}\right)+c \overline{\delta t}_{r}-c \overline{\delta t}^{s} \\
& +m_{r}^{s} T_{r}+\bar{n}_{r, I F}^{s}+\xi_{r, I F}^{s}
\end{aligned}\right.
$$

Among them,

$$
\left\{\begin{aligned}
c \overline{\delta t}_{r} & =c \delta t_{r}+B_{r, I F_{12}} \\
c \overline{\delta t} & =c \delta t^{s}+B_{I F_{12}}^{s} \\
\zeta_{r, I F}^{s y s} & =\alpha_{12} \zeta_{r}^{s y s}+\beta_{12} \zeta_{r}^{s y s} \\
\bar{n}_{r, I F}^{s} & =\lambda_{I F}\left(N_{r, 1}^{s}+\frac{f_{2}\left(N_{r, 1}^{s}-N_{r, 2}^{s}\right)}{f_{1}-f_{2}}\right) \\
& +b_{r, I F}-b_{I F}^{s}-B_{r, I F_{12}}+B_{I F_{12}}^{s}
\end{aligned}\right.
$$

where $\boldsymbol{u}_{r}^{s}=\left[\begin{array}{lll}u_{x} & u_{y} & u_{z}\end{array}\right]^{T}$ is the line-of-sight vector, $\boldsymbol{\Phi}\left(t_{0}, t\right)^{s}$ is the state transition matrix from the initial state to the current state, $\boldsymbol{x}_{0}^{s}$ is the satellite initial state parameters as well as dynamic model parameters, and $\boldsymbol{x}_{r}$ is the station positions. $\zeta_{r}^{s y s}$ denotes the inter-system bias. It is assumed that $\zeta_{r}^{s y s}$ is relative to GPS, and sys denotes other GNSS systems instead of GPS. The other terms in the ambiguity parameter are

$$
\left\{\begin{array}{l}
\lambda_{I F}=c /\left(f_{1}+f_{2}\right) \\
b_{r, I F}=c\left(f_{1} b_{r, 1}-f_{2} b_{r, 2}\right) /\left(f_{1}^{2}-f_{2}^{2}\right) \\
b_{I F}^{s}=c\left(f_{1} b_{1}^{s}-f_{2} b_{2}^{s}\right) /\left(f_{1}^{2}-f_{2}^{2}\right)
\end{array}\right.
$$

The hardware delay of the IF model is absorbed by the re-parameterized receiver clock offset $\overline{\delta t_{r}}$, satellite clock offset $\overline{\delta t}^{s}$, and ambiguity parameter $\bar{n}_{r, I F}^{s}$.

For the UC model, Eq. (1) can be re-parameterized as 


$$
\left\{\begin{array}{c}
P_{r, i}^{s}=\boldsymbol{u}_{r}^{s} \cdot\left(\boldsymbol{\Phi}\left(t_{0}, t\right)^{s} \cdot \boldsymbol{x}_{0}^{s}-\boldsymbol{x}_{r}\right)+c \overline{\delta t}_{r} \\
-c \overline{\delta t}^{s}+m_{r}^{s} T_{r}+\gamma_{i} \bar{I}_{r, 1}^{s}+\zeta_{r}^{s y s}+\varepsilon_{r, i}^{s} \\
L_{r, i}^{s}=\boldsymbol{u}_{r}^{s} \cdot\left(\boldsymbol{\Phi}\left(t_{0}, t\right)^{s} \cdot \boldsymbol{x}_{0}^{s}-\boldsymbol{x}_{r}\right)+c \overline{\delta t}_{r} \\
-c \overline{\delta t}+m_{r}^{s} T_{r}-\gamma_{i} \bar{I}_{r, 1}^{s}+\bar{n}_{r, i}^{s}+\xi_{r, i}^{s}
\end{array}\right.
$$

Among them,

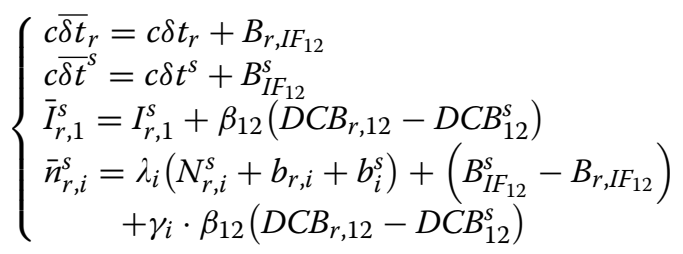

The hardware delay is absorbed by the receiver clock offset $\overline{\delta t}_{r}$, the satellite clock offset $\overline{\delta t}^{s}$, the ionospheric delay $\bar{I}_{r, 1}^{s}$, and the ambiguity parameter $\bar{n}_{r, i}^{s}$. Compared with the IF model, the UC model additionally estimates slant delay ionospheric parameters, and the ambiguity parameter absorbs the bias from the ionospheric compensation $\gamma_{i} \beta_{12}\left(D C B_{r, 12}-D C B_{12}^{s}\right)$. The estimated ionospheric delay is biased by the DCB parameters of the receiver and satellite. Hence, if the ionospheric product needs to be derived, the further separation between ionospheric delay and DCB needs to be processed. The strategy maybe like the derivation of ionospheric product for UC precise point positioning (Xiang et al. 2019).

\section{Ambiguity resolution and equivalence verification}

The integer AR can significantly improve the precision of satellite POD. The AR method of the IF strategy is omitted here. The strategy used for the UC model is essentially the same as that used for the IF model. First, Hatch-Melbourne-Wübbena combination observations are used

$$
\frac{1}{f_{1}-f_{2}}\left(f_{1} L_{1}-f_{2} L_{2}\right)-\frac{1}{f_{1}+f_{2}}\left(f_{1} P_{1}+f_{2} P_{2}\right)=\lambda_{w} N_{w}+\text { bias }
$$

where $N_{w}=\bar{N}_{r, 1}^{s}-\bar{N}_{r, 2}^{s}$ is the wide lane (WL) ambiguity, and $\lambda_{w}=c /\left(f_{1}-f_{2}\right)$ is the WL wavelength, which is approximately $0.86 \mathrm{~m}$ for GPS (L1 and L2) and $1.02 \mathrm{~m}$ for BDS (B1I and B3I). The double-difference (DD) operation is performed to obtain the WL DD ambiguity $N_{(r, q), w}^{(s, l)}$ and the standard deviation (STD) $\sigma_{w, i}$, where $s$ and $l$ denote two satellites, and $r$ and $q$ denote two stations. According to the probability determination function, the integer value $\hat{N}_{(r, q), w}^{(s, l)}$ is obtained. The ambiguity vector and covariance matrix of the float solution in unit of meters are $\boldsymbol{n}=\left[\begin{array}{ll}\boldsymbol{n}_{1} & \boldsymbol{n}_{2}\end{array}\right]^{T}$ and $\boldsymbol{\Omega}_{\boldsymbol{n}}$, where $\boldsymbol{n}_{1}$ and $\boldsymbol{n}_{2}$ denote ambiguities at the first and second frequencies, respectively. Then, the narrow lane (NL) DD ambiguity and its STD can be obtained

$$
\left\{\begin{array}{l}
N_{(r, q), n}^{(s, l)}=\frac{f_{1}+f_{2}}{c}\left[\alpha_{12} \mathbf{d} \beta_{12} \mathbf{d}\right] n-\frac{f_{1}}{f_{1}-f_{2}} \hat{N}_{(r, q), w}^{(s, l)} \\
\sigma_{n}^{D}=\frac{f_{1}+f_{2}}{c} s q r t\left(\left[\alpha_{12} \mathbf{d} \beta_{12} \mathbf{d}\right] \boldsymbol{\Omega}_{\boldsymbol{n}}\left[\alpha_{12} \mathbf{d} \beta_{12} \mathbf{d}\right]^{T}\right)
\end{array}\right.
$$

where $\mathbf{d}$ is the mapping vector from UD to DD at one frequency. The ionospheric compensation $\gamma_{i} \beta_{12}\left(D C B_{r, 12}-D C B_{12}^{s}\right)$ of the ambiguity vector $\boldsymbol{n}$ is removed by using the coefficients of $\alpha_{12}$ and $\beta_{12}$. This means that there is no difference for the float IF DD ambiguity between the IF and UC strategies. In addition, the DD operation is formed by two stations and two satellites with a baseline length, so it is inevitable that the error exists in the propagation path, especially for the ionospheric residual. Hence, when using the IF coefficients for the NL DD ambiguity resolution, the ionospheric residual of the first-order item can be removed. Then, the derived integer NL DD ambiguity is identical for the IF and UC strategies. Hence, the AR solutions of the IF and UC strategies are theoretically equivalent.

\section{Results and analysis}

Currently, few MGEX stations can receive the new signals B1C, B2a, and B2b of BDS-3. Most stations can only receive the old signals $\mathrm{B} 1 \mathrm{I}$ and B3I. Therefore, observations of $\mathrm{B} 1 \mathrm{I} / \mathrm{B} 3 \mathrm{I}$ are used for POD of BDS-3. The deployment of the BDS-3 full constellation has not been completed, and available satellites of the BDS-3 system include 18 medium Earth orbit (MEO) satellites. Hence, the POD experiments of the BDS-3 single system and BDS-3/GPS dual system have been processed. The POD period was 25 days from day of year (DOY) 195 to 219 of 2019. Observations were from 56 multi-GNSS experiment (MGEX) stations, as shown in Fig. 1. The processing strategy for POD is listed in Table 1. Six POD solutions were obtained to validate the accuracy of POD adequately.
S1: BDS-3 IF POD with 1d arc
S2: BDS-3 UC POD with 1d arc
S3: GPS IF POD with $1 \mathrm{~d}$ arc
S4: GPS UC POD with 1d arc
S5: GPS/BDS-3 IF POD with 1d arc
S6: GPS/BDS-3 UC POD with $1 \mathrm{~d}$ arc

The orbit-parameters are estimated with the tropospheric delay, Earth orientation parameter, and station coordinates simultaneously for each POD solution.

\section{POD results}

Three methods are used to assess the orbit accuracy, divided into external and internal checks. The external orbit checks are compared with external POD products (WUM products for BDS satellites and IGS final product 


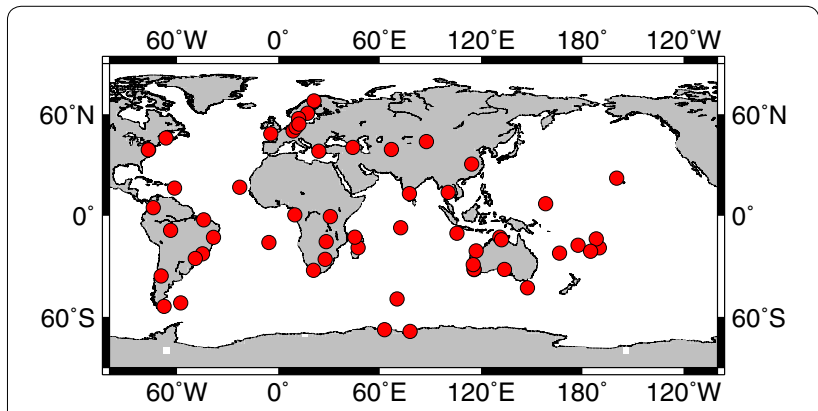

Fig. 1 Distribution of 56 stations used for POD (all stations can receive $B D S-3$ signals)

for GPS satellites) and SLR residuals validation. The internal orbit check uses the orbit boundary discontinuity (OBD) method.

Table 1 Processing strategy for POD

\begin{tabular}{|c|c|}
\hline Item & Description \\
\hline $\begin{array}{l}\text { Observation } \\
\text { data }\end{array}$ & $\begin{array}{l}\text { Undifferenced GPS L1/L2 and BDS-3 B1I/B3I data with } \\
\text { sampling rate of } 30 \mathrm{~s}\end{array}$ \\
\hline $\begin{array}{l}\text { Phase center } \\
\text { corrections } \\
\text { (PCCs) }\end{array}$ & $\begin{array}{l}\text { Navigation satellites and stations: igs 14.atx, where } \\
\text { the PCO of BDS-3 satellites is recommended by IGS. } \\
\text { The PCO of stations for BDS-3 B1I/B3I signals use the } \\
\text { values of GPS L1/L2 frequency }\end{array}$ \\
\hline $\begin{array}{l}\text { Elevation cut- } \\
\text { off angle }\end{array}$ & $10^{\circ}$ \\
\hline $\begin{array}{l}\text { Tropospheric } \\
\text { delay }\end{array}$ & $\begin{array}{l}\text { Prior model correction + parameter estimation, one set } \\
\text { of wet delay parameters in the zenith direction is esti- } \\
\text { mated every } 2 \text { h, one set of horizontal gradients per } \\
\text { day; the prior model uses the Saastamoinen model, } \\
\text { and the mapping function is the GMF }\end{array}$ \\
\hline $\begin{array}{l}\text { Ambiguity } \\
\text { parameter }\end{array}$ & DD ambiguity constraint \\
\hline $\begin{array}{l}\text { Satellite clock } \\
\text { offset }\end{array}$ & $\begin{array}{l}\text { White noise (selecting a ground station as reference } \\
\text { clock) }\end{array}$ \\
\hline $\begin{array}{l}\text { POD sampling } \\
\text { rate }\end{array}$ & $300 s$ \\
\hline $\begin{array}{l}\text { Station coordi- } \\
\text { nate }\end{array}$ & $\begin{array}{l}\text { Prior constraints of each component are added for the } \\
\text { core stations of IGS14 with an uncertainty of } 0.02 \mathrm{~m} \\
\text { and for other stations with an uncertainty of } 1000 \mathrm{~m} \\
\text { (Ruan and Wei 2019) }\end{array}$ \\
\hline Earth gravity & EGM 2008 \\
\hline $\begin{array}{l}\text { N-body gravi- } \\
\text { tation }\end{array}$ & JPL DE405 \\
\hline Solid tide & IERS conventions 2003 \\
\hline Relativity effect & IERS conventions 2003 \\
\hline $\begin{array}{l}\text { Earth orienta- } \\
\text { tion param- } \\
\text { eter }\end{array}$ & $\begin{array}{l}\text { Pole coordinates and the drifts, UT1, and length-of- } \\
\text { day. The prior values are from the IERS-released file } \\
\text { finals2000A.data }\end{array}$ \\
\hline $\begin{array}{l}\text { Solar radiation } \\
\text { pressure }\end{array}$ & ECOM 5 parameters \\
\hline Antenna thrust & Considered for GPS \\
\hline $\begin{array}{l}\text { Earth radiation } \\
\text { pressure }\end{array}$ & Considered for GPS \\
\hline
\end{tabular}

1. Comparison with external POD products

Figure 2 shows the orbit comparison results of each POD arc for BDS-3 satellites. The orbit accuracies of the IF and UC strategies are essentially the same, although the difference in S1/S2 can reach the centimeter level on some days. The single-system (S1/S2) and dual-system (S5/S6) results show that the orbit accuracy of BDS-3 satellites is improved when GPS satellites are added. Figure 3 shows the orbit results of GPS satellites. Compared with BDS-3, the difference between the IF and UC strategies is marginal. Weiss et al. (2017) showed that approximately 60 ground stations are sufficient to obtain high-precision orbits. The accuracies of the GPS single system (S3/S4) and dual system (S5/S6) are almost the same, indicating that the GPS orbit has been processed very well.

Figure 4 shows the average orbit accuracy of each BDS-3 satellite compared with WUM products for different POD solutions, where satellite identification uses the means of the pseudo-random noise (PRN). Except for the C35-C37 satellites, the accuracies of other BDS-3 satellites are comparable. The reason is that $\mathrm{C} 35-\mathrm{C} 37$ have fewer visible stations (fewer than 15), which seriously affects the product precision. The number of visible stations for the C19-C22 and C28 satellites is approximately 50; for the C23-C27, C29, C30, C32, and C34 satellites, the number is about 30 . Figure 5 shows the orbit accuracy of each GPS satellite. The results of IF and UC are essentially the same for each satellite. The dual-system results are better in general, but there are also a few satellites for which the results are worse than the single-system results.

Figure 6 shows plots of the RMS and STD of GPS clocks of each POD arc, showing that the clock accuracies of IF and UC solutions are essentially the same,

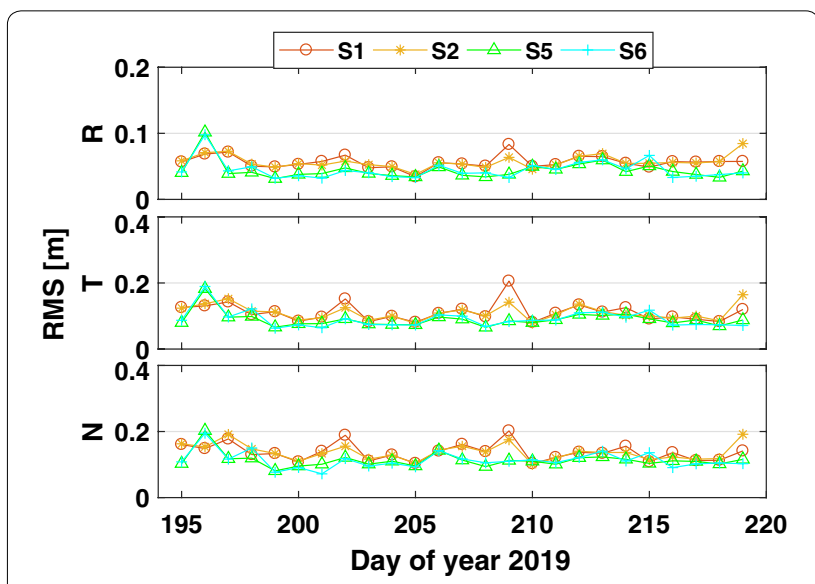

Fig. 2 Average orbit accuracy of each POD arc of BDS-3 


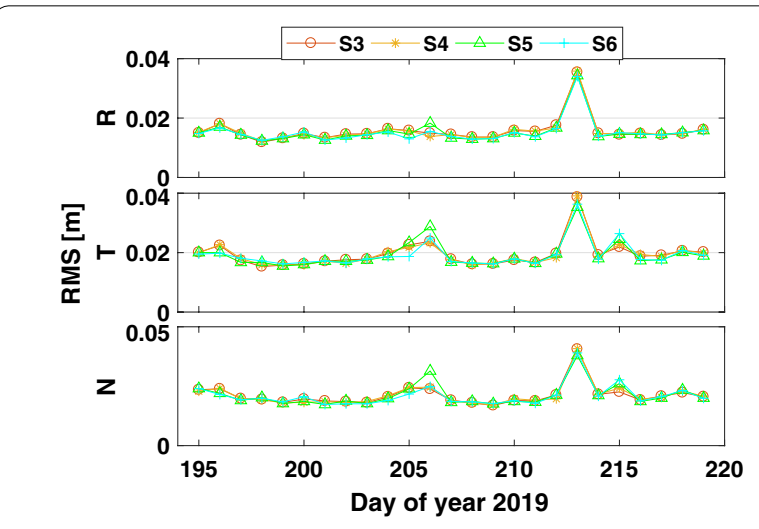

Fig. 3 Average orbit accuracy of each POD arc of GPS

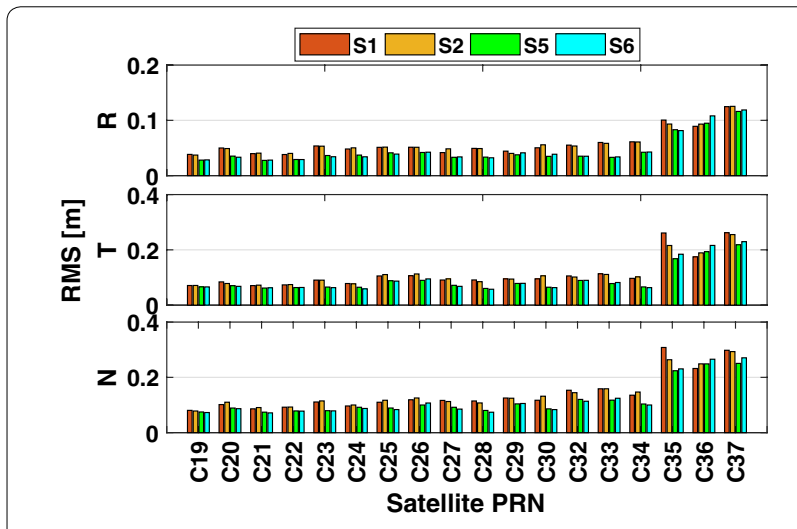

Fig. 4 Average orbit accuracy of each BDS-3 satellite

regardless of the single or dual systems. Figure 7 shows the clock results of each GPS satellite. The clock accuracies among different satellites differ significantly. This may be because of the long GPS in-orbit time, the age of satellite components, and the differences of GPS atomic clock types.

Figure 8 shows the clock STD of each BDS satellite for the S1-S4 solutions. The difference between the IF and UC solutions is small, and the clock accuracy of the dual system is better than that of the single system. Similarly, the C35-C37 clock accuracy is poor because of the small number of visible stations. The C25-C30, C34, and C35 satellites carry passive hydrogen atomic clocks, whereas the remaining satellites carry rubidium atomic clocks (Jia et al. 2019). The clock STD difference between the two clock types is not evident.

Table 2 summarizes the average RMS of the orbits in the R, T, N, and three-dimensional (3D) directions, clock RMS and STD, and data usage for the six solutions during the whole period. In addition, the difference
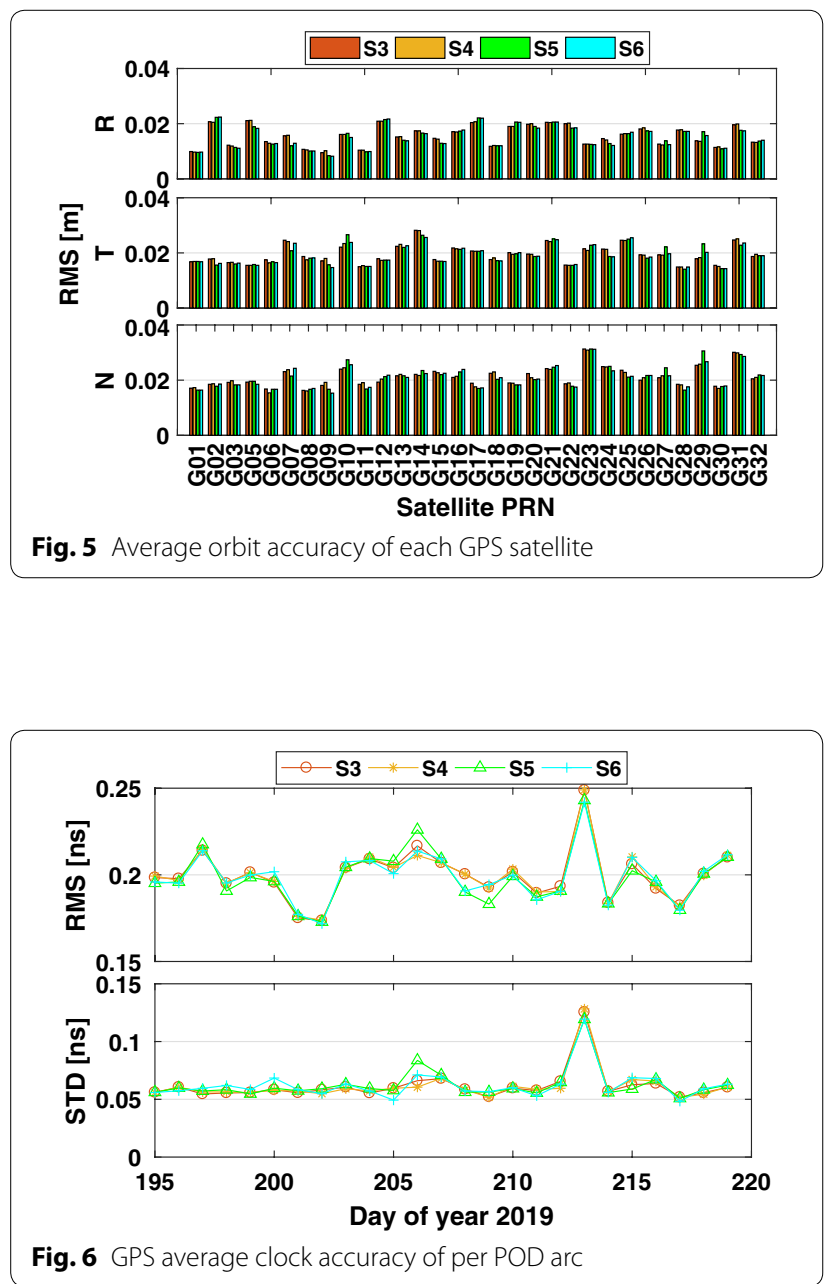

percentage of the UC compared with IF strategy is given. For GPS satellites, the POD accuracy is excellent. Compared with the IGS final product, the orbit accuracies of single and dual systems in the $\mathrm{R}, \mathrm{T}, \mathrm{N}$, and $3 \mathrm{D}$ directions are approximately $1.5,1.9,2.1$, and $3.3 \mathrm{~cm}$, respectively. The clock STD is approximately 0.06 ns. The difference between IF and UC is less than $1 \%$. The data usage rate is essentially the same, which verifies that IF and UC results are consistent. The following are possible reasons for the difference.

1. The noise levels for the IF and UC strategies are different, where the IF strategy is amplified about three times compared with the UC strategy. This may be the most important factor inducing the difference in the results, because the following two reasons can be overcome by a data quality check.

2. The difference in data usage is another possible reason. Because POD requires multiple iterations, each iteration requires the editing of residuals and removal 


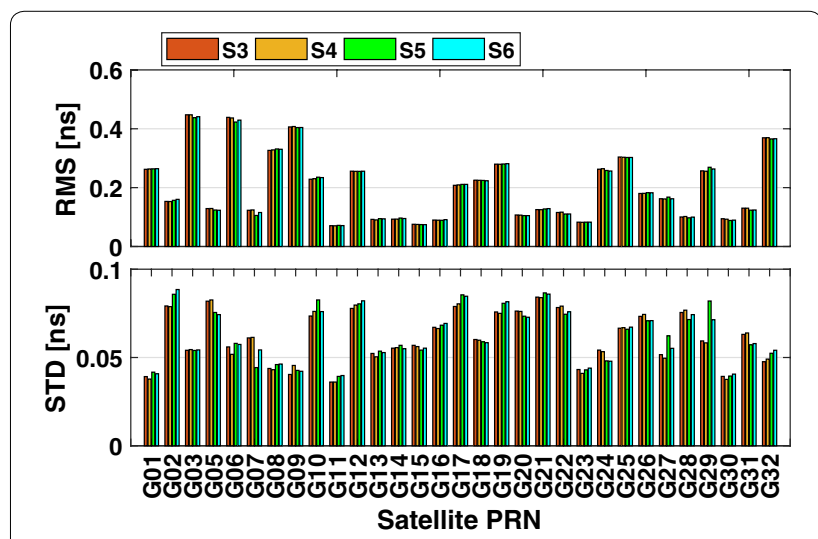

Fig. 7 GPS average clock accuracy of per satellite

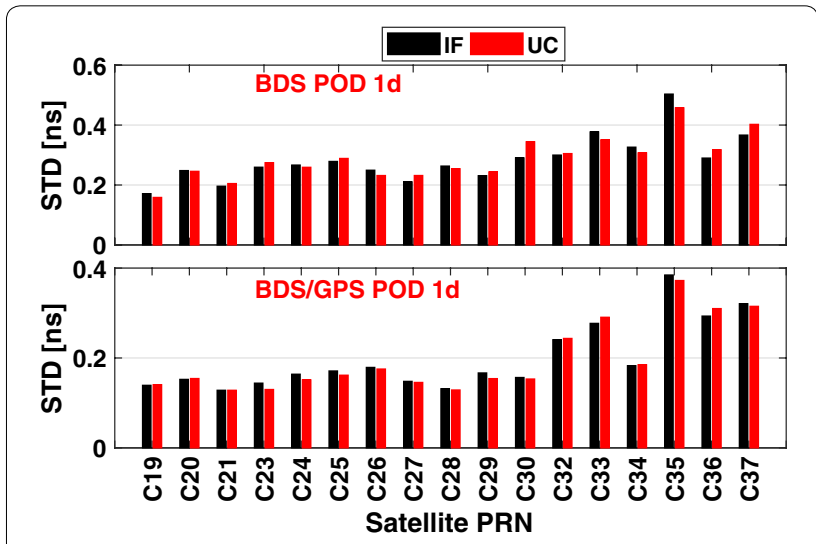

Fig. 8 Average clock accuracy of each BDS-3 satellite (S1-S4) of observations exceeding the threshold. Evidently, because of the inconsistent noise levels of the two strategies, difference in available observations inevitably occurs.

3. The difference in ambiguity fixing rate is a third possible reason. Residual editing further affects the baseline networking and the selection of independent baselines for AR. As a consequence, there may be differences in the number of fixed baselines and the selected independent baseline. This influences the AR results.

For the BDS results, the accuracy of the dual system is better than that of the single system, where the orbit RMS in the R, T, N, and 3D directions is approximately 4.4, 8.9, 11.3, and $15.2 \mathrm{~cm}$, respectively, and the clock STD is approximately 0.19 ns. The BDS-3 clock RMS for the dual-system solution is not given because a GPS satellite was selected as the reference clock. The orbit accuracy of the dual system improved by $1-2 \mathrm{~cm}$ compared with the single system in three directions, and the clock STD improved by $0.05 \mathrm{~ns}$. The results of the IF and UC strategies are essentially the same for BDS-3 satellites, but the difference is slightly larger than that for GPS. The reason may be that the removed observations are not consistent during the residual editing, resulting in differences in parameter adjustment and ambiguity resolution. The difference in data usage between the IF and UC strategies is small-less than $0.1 \%$ for both GPS and BDS. The accuracy of the orbit and clock of BDS-3 has a certain gap compared with GPS. The reason may be related to the incomplete constellation, the imperfection of force, the measurement models, and spare tracking stations for BDS-3 satellites.

Table 2 Orbit and clock accuracy and data usage rate compared with WUM (BDS-3) and IGS (GPS) products

\begin{tabular}{|c|c|c|c|c|c|c|c|}
\hline \multirow[t]{2}{*}{ Solution } & \multicolumn{4}{|c|}{ Orbits (m) } & \multicolumn{2}{|c|}{ Clocks (ns) } & \multirow[t]{2}{*}{ Usage rate (\%) } \\
\hline & $\mathbf{R}$ & $T$ & $\mathbf{N}$ & 3D & RMS & STD & \\
\hline S3 & 0.0157 & 0.0195 & 0.0212 & 0.0335 & 0.1997 & 0.0614 & 98.92 \\
\hline S4 & 0.0157 & 0.0195 & 0.0212 & 0.0335 & 0.1998 & 0.0613 & 98.91 \\
\hline S3/S4 & $0.03 \%$ & $0.23 \%$ & $-0.19 \%$ & $0.00 \%$ & $-0.03 \%$ & $0.06 \%$ & $0.01 \%$ \\
\hline S5-G & 0.0153 & 0.0193 & 0.0212 & 0.0333 & 0.1986 & 0.0625 & 98.89 \\
\hline S6-G & 0.0152 & 0.0192 & 0.0210 & 0.0330 & 0.1990 & 0.0623 & 98.88 \\
\hline S5/S6 & $0.94 \%$ & $0.56 \%$ & $1.08 \%$ & $0.94 \%$ & $-0.21 \%$ & $0.24 \%$ & $0.00 \%$ \\
\hline $\mathrm{S} 5-\mathrm{C}$ & 0.0441 & 0.0891 & 0.1129 & 0.1522 & & 0.1951 & 98.92 \\
\hline S6-C & 0.0448 & 0.0905 & 0.1129 & 0.1534 & & 0.1926 & 98.96 \\
\hline S5/S6 & $-1.58 \%$ & $-1.63 \%$ & $0.01 \%$ & $-0.79 \%$ & & $1.26 \%$ & $-0.04 \%$ \\
\hline S1 & 0.0565 & 0.1114 & 0.1374 & 0.1879 & 1.0028 & 0.2584 & 99.05 \\
\hline S2 & 0.0570 & 0.1104 & 0.1378 & 0.1876 & 1.0012 & 0.2612 & 99.12 \\
\hline S1/S2 & $-0.75 \%$ & $0.90 \%$ & $-0.27 \%$ & $0.20 \%$ & $0.16 \%$ & $-1.08 \%$ & $-0.06 \%$ \\
\hline
\end{tabular}




\section{Orbit boundary discontinuities}

OBD is used to evaluate the orbit accuracy further. Figure 9 shows the BDS-3 orbit accuracy of each POD arc of the six solutions. The OBD of $1 \mathrm{~d}$ solutions is on the order of $1 \mathrm{dm}$ in the $\mathrm{R}$ direction, and $1-2 \mathrm{dm}$ in the $\mathrm{T}$ and $\mathrm{N}$ directions. The orbit accuracies of the IF and UC strategies are essentially the same. Figure 10 shows the results of each BDS-3 satellite, and it shows that the accuracy of OBD for the dual-system results is better. The IF and UC results are slightly different.

Figure 11 shows the GPS OBD. The IF and UC results in the cases of single- and dual-system solutions are essentially the same, with the OBD being approximately $5 \mathrm{~cm}$ in three directions. Figure 12 shows the average accuracy of the OBD of each GPS satellite, and the difference between the IF and UC strategies is small.

Table 3 summarizes the overall orbit accuracy of OBD. GPS has superior consistency. The difference in OBD accuracy between IF and UC is on the order of millimeters, and the average 3D accuracy is approximately $6 \mathrm{~cm}$. However, the accuracy of BDS-3 is worse than that of GPS, with the 3D RMS being approximately $2 \mathrm{dm}$. There is a difference of $1-2 \mathrm{~cm}$ in the $3 \mathrm{D}$ direction of the IF and UC strategies for BDS-3. The BDS-3 accuracy of the dual system is better than that of the single system, improving by $1-3 \mathrm{~cm}$ in each direction.

\section{SLR validation}

Considering that the WUM product precision of BDS-3 satellites is unknown, another external means, i.e., SLR residuals, is used to check the orbit accuracy of BDS satellites further. The laser retroreflector array offset reference to the center of the satellite mass can be found in the work of Xu et al. (2019) for four BDS-3 MEO satellites tracked by the International Laser Ranging Service. Figure 13 shows the SLR residuals of each POD arc for the six solutions. The residuals of the dual system are smaller than those of the single system. Moreover, the SLR residuals follow a zero-mean distribution. Table 4 summarizes the average offset, STD, RMS, and number of normal points (NPs). Some NPs with residual values greater than $2 \mathrm{~m}$ or more than five times the median error are excluded. The mean offset of the SLR residuals is within $3 \mathrm{~cm}$, and, for the C29 and C30 satellites, it is slightly larger. In general, the dual-system results are better than the results of the single system, improving by approximately $1-2 \mathrm{~cm}$. The reason may be that, with the addition of GPS, the accuracy of common parameters can be improved. In the case of single-system 1d POD, the available observations with only 18 satellites and 56 stations may be insufficient. There are some differences

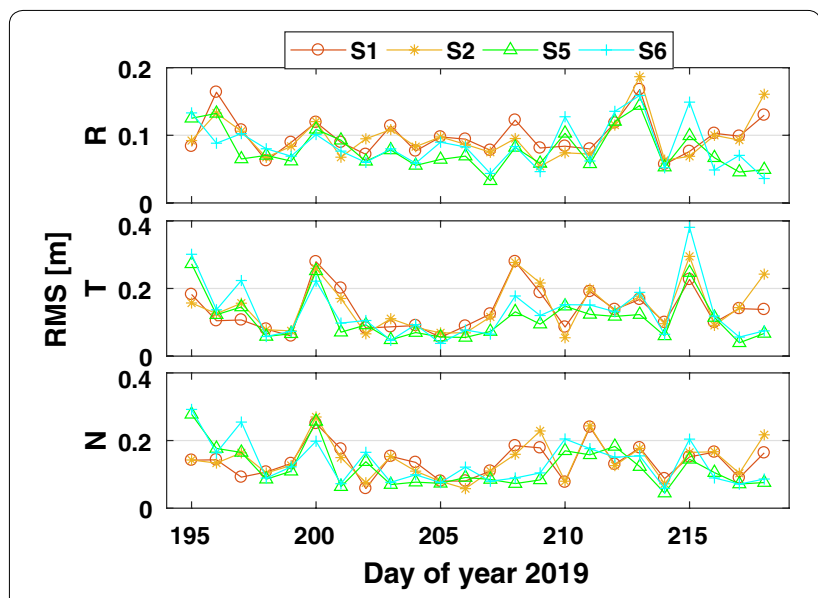

Fig. 9 BDS-3 OBD of each POD arc
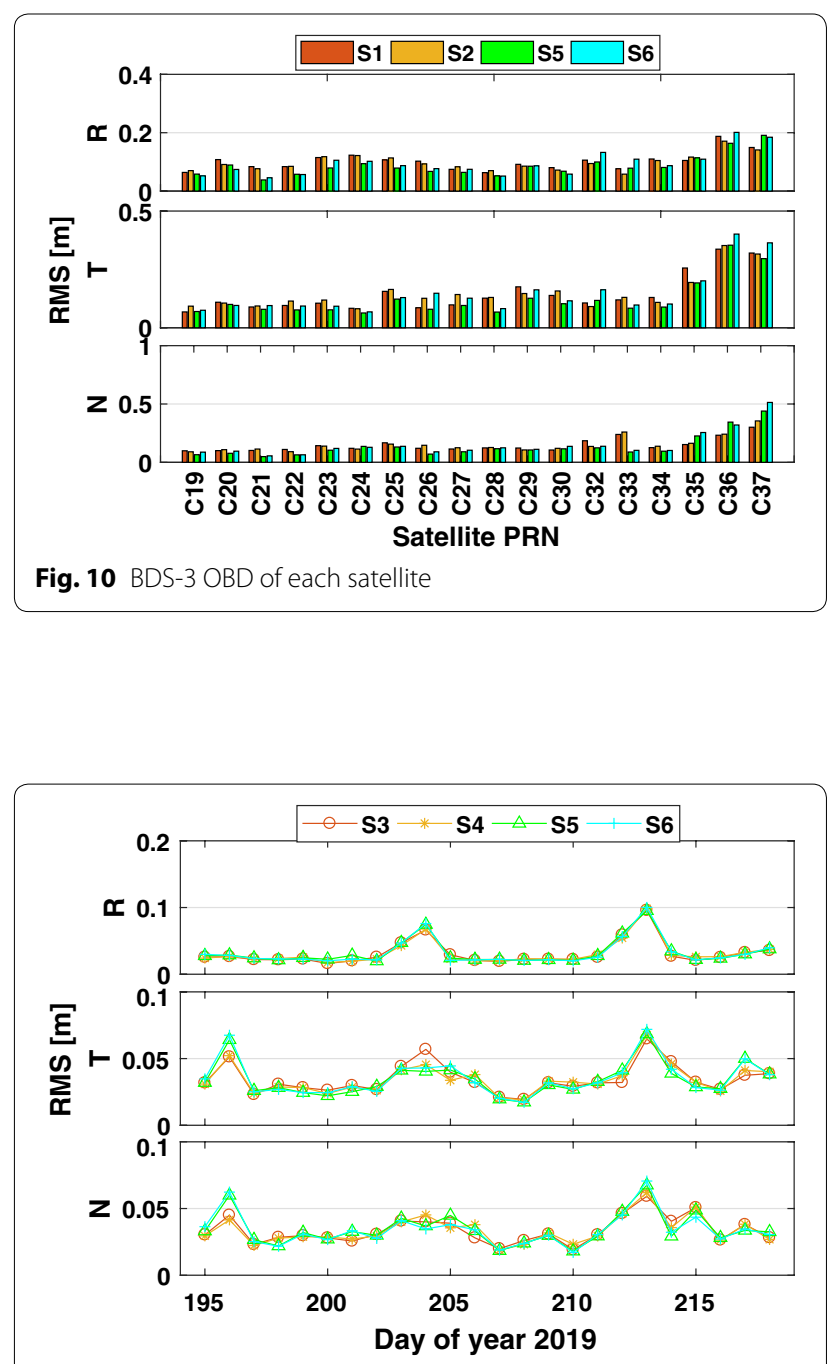

Fig. 11 GPS OBD of each POD arc 


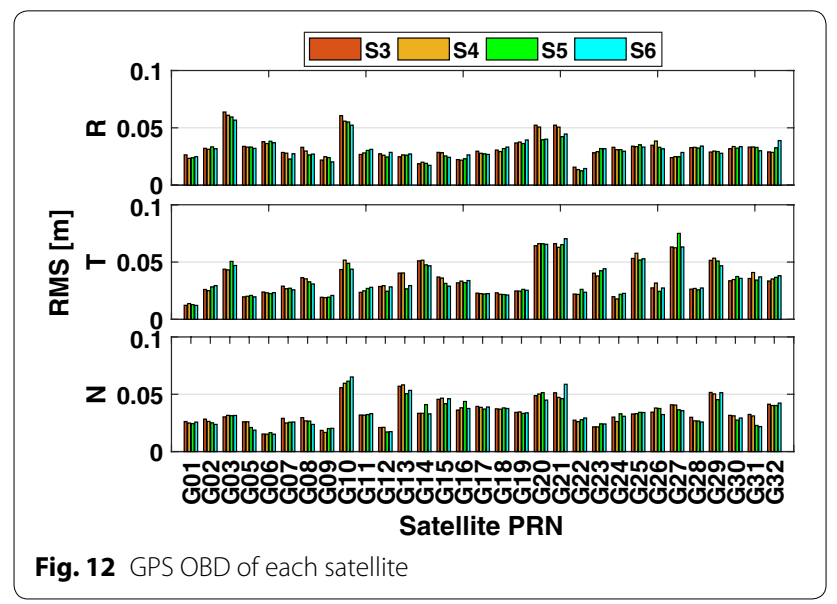

between the IF and UC strategies, with the magnitude being at a millimeter level. The RMS of SLR residuals is approximately $4 \mathrm{~cm}$, which is in the same magnitude as that in Xu et al. (2019).
Table 3 OBD of BDS-3 and GPS

\begin{tabular}{lllll}
\hline Solution & \multicolumn{4}{l}{ Orbits $(\mathbf{m})$} \\
\cline { 2 - 5 } & $\mathbf{R}$ & $\mathbf{T}$ & $\mathbf{N}$ & 3D \\
\hline S3 & 0.0326 & 0.0345 & 0.0345 & 0.0679 \\
S4 & 0.0322 & 0.0352 & 0.034 & 0.0677 \\
S5-G & 0.0313 & 0.0348 & 0.0335 & 0.0665 \\
S6-G & 0.0316 & 0.0345 & 0.0336 & 0.0663 \\
S5-C & 0.0791 & 0.1103 & 0.1208 & 0.2083 \\
S6-C & 0.0848 & 0.1314 & 0.1333 & 0.2356 \\
S1 & 0.0986 & 0.1374 & 0.1377 & 0.2529 \\
S2 & 0.0958 & 0.1442 & 0.1421 & 0.2607 \\
\hline
\end{tabular}

\section{POD calculation time}

To validate further the computation efficiency for the IF and UC strategies, Table 5 shows the cost time of the six POD solutions for the first iteration and total POD processing. The statistics may not be highly strict, because a few POD tests are executed in parallel. The computation time of the UC strategy (S2, S4, and S6) is greater than

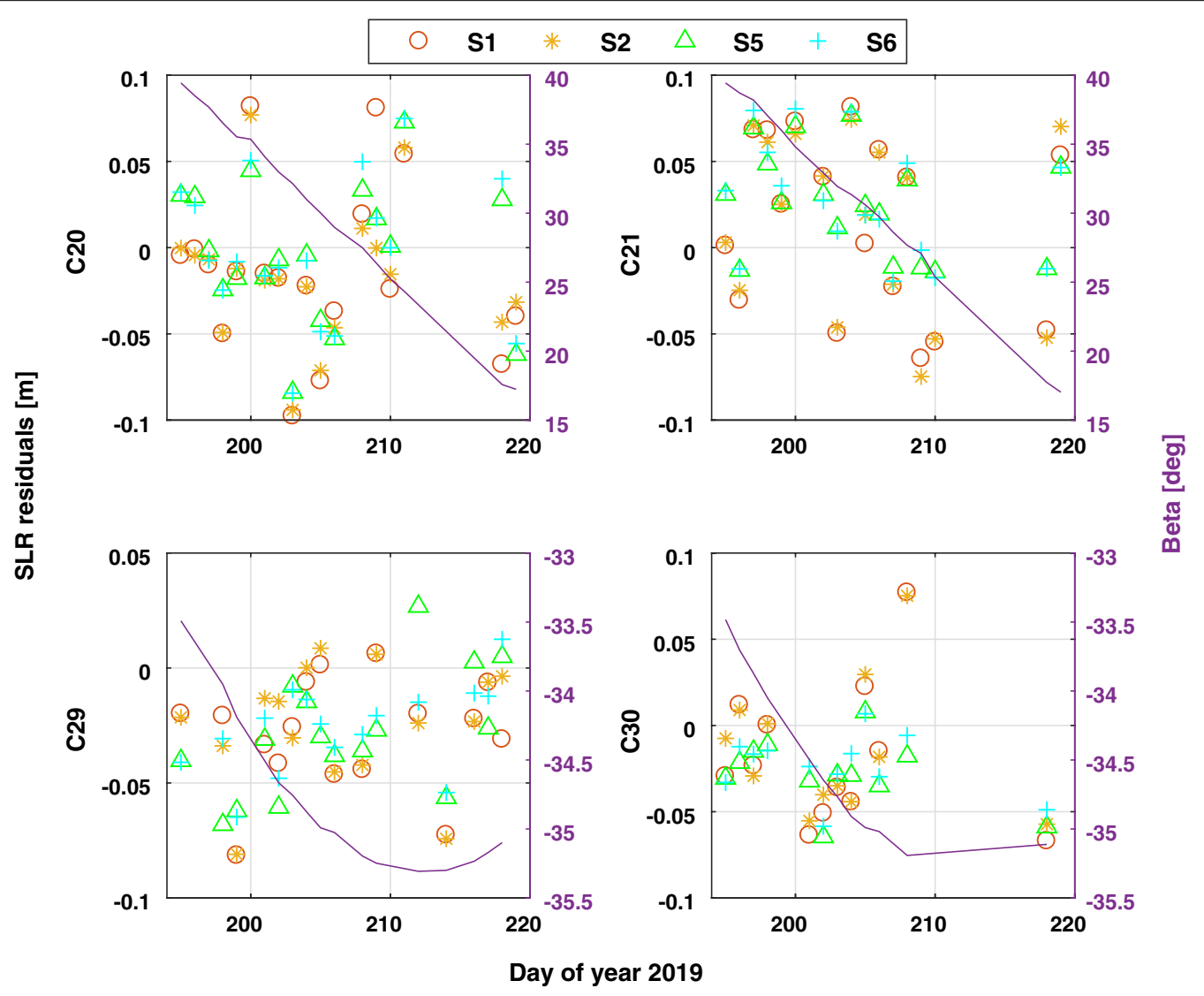

Fig. 13 SLR residual results for four BDS-3 satellites 
Table 4 SLR check results $/ \mathrm{m}$

\begin{tabular}{|c|c|c|c|c|c|}
\hline & Solution & \#NP & Offset & STD & RMS \\
\hline \multirow[t]{4}{*}{$C 20$} & S1 & 162 & -0.018 & 0.054 & 0.057 \\
\hline & S2 & 162 & -0.021 & 0.044 & 0.049 \\
\hline & S5 & 162 & -0.009 & 0.038 & 0.039 \\
\hline & S6 & 162 & -0.008 & 0.042 & 0.042 \\
\hline \multirow[t]{4}{*}{ C21 } & S1 & 113 & 0.011 & 0.068 & 0.069 \\
\hline & S2 & 113 & 0.013 & 0.070 & 0.071 \\
\hline & S5 & 113 & 0.022 & 0.045 & 0.050 \\
\hline & S6 & 113 & 0.024 & 0.050 & 0.056 \\
\hline \multirow[t]{4}{*}{ C29 } & S1 & 74 & -0.033 & 0.033 & 0.046 \\
\hline & S2 & 74 & -0.032 & 0.033 & 0.046 \\
\hline & S5 & 74 & -0.031 & 0.034 & 0.046 \\
\hline & S6 & 74 & -0.030 & 0.026 & 0.039 \\
\hline \multirow[t]{4}{*}{ C30 } & S1 & 64 & -0.028 & 0.041 & 0.050 \\
\hline & S2 & 64 & -0.023 & 0.040 & 0.046 \\
\hline & S5 & 64 & -0.034 & 0.026 & 0.042 \\
\hline & S6 & 64 & -0.029 & 0.024 & 0.038 \\
\hline
\end{tabular}

that of the IF strategy (S1, S3, and S5). The first iteration time is greater by 1.0, 8.8, and $21.0 \mathrm{~min}$ for the BDS-only, GPS-only, and GPS/BDS POD; correspondingly, the total POD time is greater by $3.1,49.7$, and $137.2 \mathrm{~min}$, respectively. The reason is that the UC strategy needs additionally to estimate the slant ionospheric delay and the double ambiguity parameters. An improved parameter elimination method is used for the ionospheric delay; otherwise, the computing time of the UC strategy could be approximately five times greater (Zeng et al. 2019b). Furthermore, the ambiguity parameters of the whole POD arc are added to the least square adjustment, and no parameter elimination method is used, because the upper triangular square root covariance update algorithm is used for ambiguity resolution (Ruan 2015). For multi-GNSS UC POD with a considerable number of satellites and stations, the problem of computation efficiency must be resolved further, especially the inversion of high dimensional ambiguity parameters. We will study the problem in future.

\section{Ambiguity fixing rate}

Figure 14 shows the ambiguity fixing rates of the GPS results. The average fixing rates of the WL and NL of the IF strategy for the dual system are approximately $96.1 \%$ and $90.8 \%$, respectively. They are slightly better than those for the single system, which are approximately $95.9 \%$ and $90.6 \%$, respectively. The fixing rates of IF and UC strategies are essentially the same, and the UC result is slightly higher by $0.1 \%$. Figure 15 is the ambiguity fixing rate of the BDS results. The fixing rate of BDS-3 is worse than that of GPS. The dual-system solution (S5/ S6) achieves the optimal fixing rate, with the WL and NL being $92.2 \%$ and $83.3 \%$, respectively. The $1 \mathrm{~d}$ solution (S1/ S2) is worse, with the WL and NL being $86.4 \%$ and $69.9 \%$ for the IF strategy, respectively. The fixing rates for the IF and UC strategies are consistent, but the differences are larger than for GPS. The most important reason is that the BDS-3 constellation is incomplete. The baseline is set up from ground stations and satellites of one GNSS system when processing AR. The number of BDS-3 satellites is evidently limited. The distribution and quality of baselines affect the fixing rate. However, the results of the dual system are significantly better than those of the single system, because the addition of GPS enhances the accuracy of the common parameter solution. In addition, the ambiguity of BDS is updated after the independent DD ambiguity set of GPS is processed. At this time, the correlation among the ambiguity parameters has been further reduced.

\section{Conclusion}

In this study, IF and UC POD observation models were analyzed. The difference between the two models after reparameterization was verified, as reflected in the ambiguity and ionosphere parameters. The difference can be eliminated when using the WL-NL AR method. Therefore, the AR solutions of the IF and UC observation models are equivalent. This study fully demonstrated the product accuracy of BDS-3 satellites using the UC method, and the single/dual-system 1d POD solutions were analyzed. Three methods were used to check the precision of the derived BDS-3 products, including comparison with WUM products, OBD, and SLR residual validation. POD results were demonstrated for many aspects, such as orbits, clocks, ambiguity fixing rate, data usage rate, and computing time. The conclusions are as follows.

1. BDS-3 products achieve good accuracy. Compared with WUM, the orbit accuracies in the $\mathrm{R}, \mathrm{T}$, and

Table 5 Computing time of the first iteration and POD/min

\begin{tabular}{lllcccc}
\hline & S1 & S2 (diff.) & S3 & S4 (diff.) & S5 & S6 (diff.) \\
\hline 1st iteration & 1.2 & $2.2(+1.0)$ & 4.6 & $13.5(+8.8)$ & 5.4 & $26.4(+21.0)$ \\
POD & 5.1 & $8.2(+3.1)$ & 30.3 & $80.0(+49.7)$ & 37.1 & $174.3(+137.2)$ \\
\hline
\end{tabular}




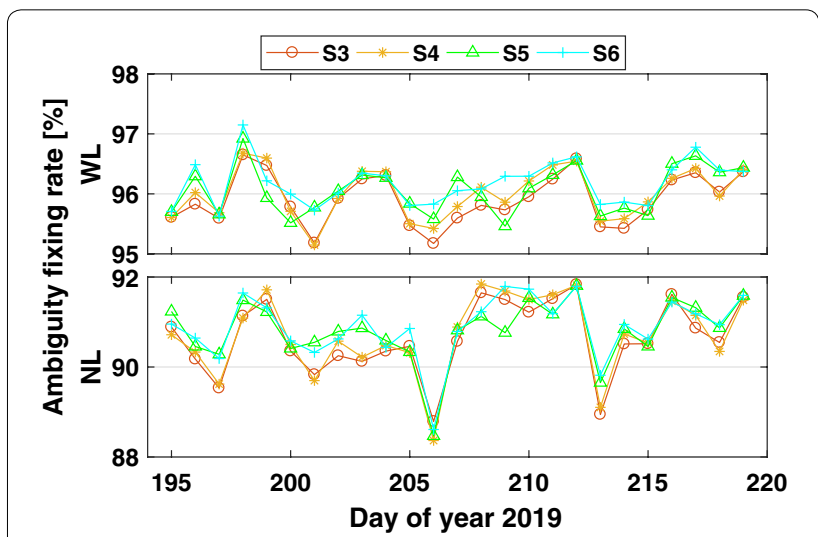

Fig. 14 GPS ambiguity fixing rate

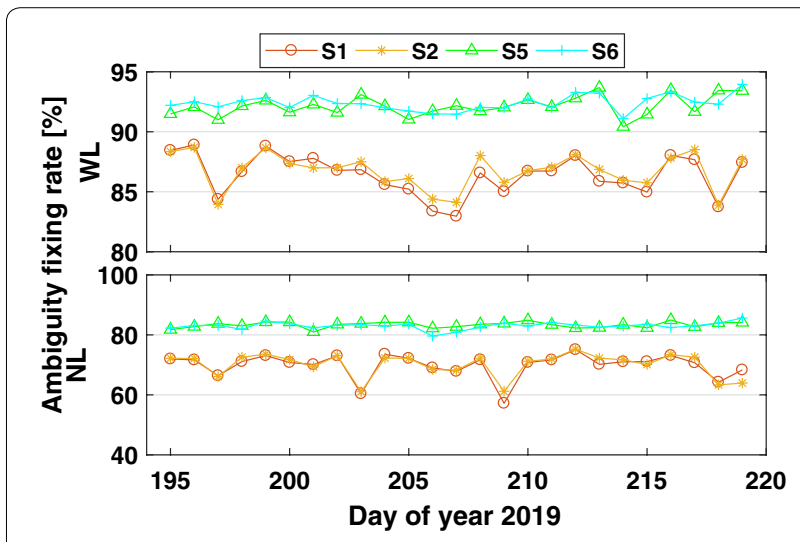

Fig. 15 BDS-3 ambiguity fixing rate

$\mathrm{N}$ directions are 4.4, 9.0, and $11.3 \mathrm{~cm}$ for the dualsystem $1 \mathrm{~d}$ solution. The clock STD is approximately $0.2 \mathrm{~ns}$, and the RMS of SLR residuals is about $4 \mathrm{~cm}$. The orbit 3D RMS of GPS is approximately $3.3 \mathrm{~cm}$ compared with IGS final products.

2. In general, the product accuracies of the IF and UC strategies are the same. The difference for orbits and clocks is within $3 \%$, whereas, for the data usage rate, it is less than $0.1 \%$. The reason is mainly the noise level of the observation model. The GPS difference is smaller than the result of BDS. The computing time of the UC strategy is greater than that of IF strategy.

\section{Acknowledgement}

IGS MGEX is gratefully acknowledged for providing data and products. We express our sincere gratitude to editors and anonymous reviewers for improving the paper.

\section{Authors' contributions}

TZ designed the research, performed the research, analyzed the data and wrote the paper. RR developed the software of satellite positioning and orbit determination system (SPODS). LS and XJ revised the research and supervised the experiments. RR and LF revised the paper. All authors read and approved the final manuscript.

\section{Funding}

The research was substantially funded by National Natural Science Foundation of China (Grant Nos. 41674016, 41874041, 41704035, 41904039) and by State Key Laboratory of Geo-Information Engineering, NO. SKLGIE2018-M-2-1.

\section{Availability of data and materials}

The GNSS data and product can be derived by the IGS with the website http:// www.igs.org.

\section{Competing interests}

The authors declare that they have no competing interests.

\section{Author details}

${ }^{1}$ Information Engineering University, Zhengzhou 450001, People's Republic of China. ${ }^{2}$ State Key Laboratory of Geo-Information Engineering, Xi'an 710054 People's Republic of China. ${ }^{3}$ Xi'an Research Institute of Surveying and Mapping, Xi'an 710054, People's Republic of China.

Received: 30 January 2020 Accepted: 6 May 2020

Published online: 22 June 2020

\section{References}

Chen, H. (2015). An efficient and unified GNSS raw data processing strategy. Ph.D. thesis, Wuhan University (in Chinese).

Gu, S., Lou, Y., Shi, C., \& Liu, J. (2015). BeiDou phase bias estimation and its application in precise point positioning with triple-frequency observable. Journal of Geodesy, 89(10), 979-992.

Guo, J. (2014). The impacts of attitude, solar radiation and function model on precise orbit determination for GNSS satellites. Ph.D. thesis, Wuhan University (in Chinese)

Guo, J., \& Geng, J. (2018). GPS satellite clock determination in case of interfrequency clock biases for triple-frequency precise point positioning. Journal of Geodesy, 92(10), 1133-1142.

Guo, S., Cai, H., Meng, Y., Geng, C., Jia, X., Mao, Y., et al. (2019). BDS-3 RNSS technical characteristics and service performance. Acta Geodaetica et Cartographica Sinica, 48(7), 810-821 (in Chinese).

He, C., Lu, X., Guo, J., Su, C., Wang, W., \& Wang, M. (2020). Initial analysis for characterizing and mitigating the pseudorange biases of BeiDou navigation satellite system. Satellite Navigation, 1(1), 1-10.

Jia, X., Zeng, T., Ruan, R., Mao, Y., \& Xiao, G. (2019). Atomic clock performance assessment of BeiDou-3 basic system with the noise analysis of orbit determination and time synchronization. Remote Sensing, 11(24), 2895.

Li, P., Zhang, X., Ge, M., \& Schuh, H. (2018). Three-frequency BDS precise point positioning ambiguity resolution based on raw observables. Journal of Geodesy, 92(12), 1357-1369.

Li, X., Ge, M., Zhang, H., \& Wickert, J. (2013). A method for improving uncalibrated phase delay estimation and ambiguity-fixing in real-time precise point positioning. Journal of Geodesy, 87(5), 405-416.

Li, X., Yuan, Y., Zhu, Y., Huang, J., Wu, J., Xiong, Y., et al. (2019). Precise orbit determination for BDS3 experimental satellites using iGMAS and MGEX tracking networks. Journal of Geodesy, 93(1), 103-117.

Pan, L., Zhang, X., Guo, F., \& Liu, J. (2019). GPS inter-frequency clock bias estimation for both uncombined and ionospheric-free combined triplefrequency precise point positioning. Journal of Geodesy, 93(4), 473-487.

Ruan, R. (2015). Ambiguity resolution for GPS/GNSS network solution implemented in SPODS. Acta Geodaetica et Cartographica Sinica, 44(2), 128-134. (in Chinese)

Ruan, R., Jia, X., Wu, X., Feng, L., \& Zhu, Y. (2014). SPODS software and its result of precise orbit determination for GNSS satellites. In China satellite navigation conference (CSNC) 2014 proceedings: Volume III (pp. 301-312). Springer, Berlin, Heidelberg.

Ruan, R., \& Wei, Z. (2019). Between-satellite single-difference integer ambiguity resolution in GPS/GNSS network solutions. Journal of Geodesy, 93(9), 1367-1379

Strasser, S., Mayer-Gürr, T., \& Zehentner, N. (2019). Processing of GNSS constellations and ground station networks using the raw observation approach. Journal of Geodesy, 93(7), 1045-1057. 
Tu, R., Zhang, P., Zhang, R., Liu, J., \& Lu, X. (2019). Modeling and performance analysis of precise time transfer based on BDS triple-frequency uncombined observations. Journal of Geodesy, 93(6), 837-847.

Weiss, J. P., Steigenberger, P., \& Springer, T. (2017). Orbit and clock product generation. In P. J. G. Teunissen \& O. Montenbruck (Eds.), Springer handbook of global navigation satellite systems (pp. 983-1010). Cham: Springer.

Xiang, Y., Gao, Y., Shi, J., \& Xu, C. (2019). Consistency and analysis of ionospheric observables obtained from three precise point positioning models. Journal of Geodesy, 93(8), 1161-1170.

Xu, X., Wang, X., Liu, J., \& Zhao, Q. (2019). Characteristics of BD3 global service satellites: $P O D$, open service signal and atomic clock performance. Remote Sensing, 11(13), 1559.

Yan, X., Huang, G., Zhang, Q., Liu, C., Wang, L., \& Qin, Z. (2019). Early analysis of precise orbit and clock offset determination for the satellites of the global BeiDou-3 system. Advances in Space Research, 63(3), 1270-1279.

Yang, Y., Gao, W., Guo, S., Mao, Y., \& Yang, Y. (2019). Introduction to BeiDou-3 navigation satellite system. Navigation, 66(1), 7-18.

Yang, Y., Mao, Y., \& Sun, B. (2020). Basic performance and future developments of BeiDou global navigation satellite system. Satellite Navigation, 1(1), 1-8.

Zeng, T., Jia, X., Sui, L., Xiao, G., Yi, T., \& Zhi, L. (2019a). Initial evaluation of beidou-3 satellite data quality and single system precise orbit determination. Journal of Geodesy and Geodynamics, 39(11), 1165-1170 (in Chinese).
Zeng, T., Sui, L., Xiao, G., Ruan, R., \& Jia, X. (2019b). Computationally efficient dual-frequency uncombined precise orbit determination based on IGS clock datum. GPS Solutions, 23(4), 105.

Zhang, Z., Li, B., Nie, L., Wei, C., Jia, S., \& Jiang, S. (2019). Initial assessment of BeiDou-3 global navigation satellite system: Signal quality, RTK and PPP. GPS Solutions, 23(4), 111

Zhang, B., Ou, J., Yuan, Y., \& Li, Z. (2012). Extraction of line-of-sight ionospheric observables from GPS data using precise point positioning. Science China Earth Sciences, 55(11), 1919-1928.

Zhao, Q., Wang, Y., Gu, S., Zheng, F., Shi, C., Ge, M., et al. (2019). Refining ionospheric delay modeling for undifferenced and uncombined GNSS data processing. Journal of Geodesy, 93(4), 545-560.

\section{Publisher's Note}

Springer Nature remains neutral with regard to jurisdictional claims in published maps and institutional affiliations.

\section{Submit your manuscript to a SpringerOpen ${ }^{\odot}$ journal and benefit from:}

- Convenient online submission

- Rigorous peer review

- Open access: articles freely available online

- High visibility within the field

- Retaining the copyright to your article

Submit your next manuscript at $\boldsymbol{\nabla}$ springeropen.com 